\title{
Linoleic acid, a dietary n-6 polyunsaturated fatty acid, and the aetiology of ulcerative colitis: a nested case-control study within a European prospective cohort study
}

\author{
The IBD in EPIC Study Investigators
}

\section{See Commentary, p 1577}

Correspondence to:

Dr A Hart, University of East Anglia, Norwich, NR4 7TJ, UK : a.hart@uea.ac.uk

$\mathrm{AH}$ is a principal investigator for the IBD in EPIC (European Prospective Investigation into Cancer and Nutrition) Study. The other collaborators and authors in this study are listed at the end of the paper.

Revised 21 May 2009 Accepted 6 June 2009

\begin{abstract}
Objective: Dietary linoleic acid, an n-6 polyunsaturated fatty acid, is metabolised to arachidonic acid, a component of colonocyte membranes. Metabolites of arachidonic acid have pro-inflammatory properties and are increased in the mucosa of patients with ulcerative colitis. The aim of this investigation was to conduct the first prospective cohort study investigating if a high dietary intake of linoleic acid increases the risk of developing incident ulcerative colitis.
\end{abstract}

Design and setting: Dietary data from food frequency questionnaires were available for 203193 men and women aged 30-74 years, resident in the UK, Sweden, Denmark, Germany or Italy and participating in a prospective cohort study, the European Prospective Investigation into Cancer and Nutrition (EPIC). These participants were followed up for the diagnosis of ulcerative colitis. Each case was matched with four controls and the risk of disease calculated by quartile of intake of linoleic acid adjusted for gender, age, smoking, total energy intake and centre.

Results: A total of 126 participants developed ulcerative colitis (47\% women) after a median follow-up of 4.0 years (range, 1.7-11.3 years). The highest quartile of intake of linoleic acid was associated with an increased risk of ulcerative colitis (odds ratio $(\mathrm{OR})=2.49,95 \%$ confidence interval $(\mathrm{Cl})=1.23$ to $5.07, \mathrm{p}=0.01$ ) with a significant trend across quartiles $(\mathrm{OR}=1.32$ per quartile increase, $95 \% \mathrm{Cl}=1.04$ to $1.66, \mathrm{p}=0.02$ for trend).

Conclusions: The data support a role for dietary linoleic acid in the aetiology of ulcerative colitis. An estimated $30 \%$ of cases could be attributed to having dietary intakes higher than the lowest quartile of linoleic acid intake.

Ulcerative colitis is a chronic condition that produces distressing symptoms, impairs patients' quality of life and is associated with an increased risk of complications such as colorectal cancer. ${ }^{1}$ The causes of ulcerative colitis are unknown although it is plausible that dietary n-6 polyunsaturated fatty acids (n-6 PUFAs) could be involved. The essential n-6 PUFA, linoleic acid, is present in many sources including red meat, various cooking oils and certain margarines. Linoleic acid undergoes metabolic conversion to a further n-6 PUFA, arachidonic acid, which is a component of colonic cell membranes. Arachidonic acid can be released from this phospholipid membrane and metabolised to pro-inflammatory eicosanoids including prostaglandin $\mathrm{E}_{2}$, leukotriene $\mathrm{B}_{4}$ and thromboxane $\mathrm{A}_{2}$. These eicosanoids are present in excess in the mucosa of patients with ulcerative colitis and in animal models of the disease. ${ }^{2-5}$ The concentrations correlate with the degree of histological inflammation $^{2}$ and medications containing 5-aminosalicylic acid inhibit their formation. ${ }^{4}$ Data from epidemiological studies are needed to examine the linoleic acid hypothesis generated from the experimental and clinical observations. Several such investigations have reported a positive association with an increased total polyunsaturated fatty acid intake. ${ }^{6-8}$ However, only one used a prospective cohort design, ${ }^{7}$ and just one, a retrospective case-control investigation, specifically investigated linoleic acid consumption. ${ }^{6}$

The aim of this study was to conduct the first prospective cohort investigation of dietary linoleic acid intake and the risk of developing incident ulcerative colitis. Participants were enrolled in the European Prospective Investigation into Cancer and Nutrition (EPIC) study ${ }^{9}$ which recruited a total of 519978 volunteers in 23 centres in 10 countries. Participants provided information on diet and lifestyle and were followed up for health endpoints including ulcerative colitis. The methodological advantages of this prospective cohort design over retrospective case-control work, are that the dietary information is more accurate, as current intake is recalled which is less affected by recall bias. Additionally, the cohort design avoids potential selection biases, as those who develop ulcerative colitis and those who remain disease-free are drawn from the same population.

\section{METHODS}

Participants were resident in seven regions in five European countries. These EPIC sub-cohorts constituted 203193 men and women in the age range 30-74 years (table 1). At recruitment, between 1991 and 1998, participants provided information on diet, physical activity, and other lifestyle factors such as smoking and alcohol intake. Diet was measured by country-specific food frequency questionnaires (FFOs) that were designed to capture local dietary habits and to give high compliance. Nutrient intake was calculated by multiplying the frequency of consumption of relevant foods by their fatty acid content as determined from national databases of food content. The dietary fatty acids which were calculated were: linoleic acid (n-6 PUFA), $\alpha$-linolenic acid, eicosapentaenoic acid, docosahexaenoic acid (n-3 PUFAs) and oleic acid (an n-9 monounsaturated fatty acid). In all 
centres, the FFQs were validated against $24 \mathrm{~h}$ recall questionnaires and most centres also compared their questionnaire data against plasma and urinary biomarkers for specific nutrients including vitamin $\mathrm{C}$, vitamin $\mathrm{E}$ and protein. ${ }^{10}{ }^{11}$ The research protocols were approved by ethics committees in each centre and all participants gave written informed consent.

Incident cases of ulcerative colitis which developed in participants who were initially free of the disease were identified by several methods. These were disease registries of inflammatory bowel disease in Italy, Sweden and Denmark, from follow-up questionnaires in Germany and in the Norfolk, UK, cohort by a combination of follow-up questionnaires and hospital inpatient and pathology databases. For each case, physicians were asked to confirm the diagnosis of ulcerative colitis according to whether there was information available from radiological, endoscopic and histological reports. Information on both the extent of colonic inflammation and the investigations used to confirm disease were collected. Prevalent cases of ulcerative colitis at recruitment were excluded as well as participants who were diagnosed less than 18 months after recruitment into the EPIC study. This helped to ensure that the dietary data reflected participants' dietary intake prior to the development of symptoms. Incident cases were identified up to the end of June 2004 for most centres.

In the analysis, using a nested case-control method within a prospective cohort study, each case was matched with four randomly selected controls (from the same centre), of the same gender, date of birth ( \pm 6 months) and date of recruitment into EPIC ( \pm 3 months). Controls were alive on the date when the matched case was diagnosed. The last two criteria ensured that the periods of follow-up for both cases and controls were similar. The dietary intakes of linoleic acid and the other fatty acids were divided into gender-specific quartiles from the distribution across both cases and controls. A multivariate analysis was performed, using unconditional logistic regression, adjusting for age at recruitment, gender, total energy intake, cigarette smoking and centre. Energy adjustment helps to account for variables which affect dietary intake, namely body mass index, metabolic rate and physical activity. Smoking was included in the analysis due to the observed inverse association with the risk of ulcerative colitis. ${ }^{12}{ }^{13}$ Only one measure of smoking was used, namely that at recruitment. Adjustment for centre helps correct for possible differences in the methodology of the food frequency questionnaires between countries. Finally, the odds ratios for the individual fatty acids were adjusted for each of the four other fatty acids, for the centres for which such complete data was available. The rationale for this was that oleic acid and $\alpha$-linolenic acid competitively inhibit the metabolism of linoleic acid and reduce the formation of arachidonic acid. Additionally, the $n-3$ polyunsaturated fatty acids eicosapentaenoic acid and docosahexaenoic acid have antiinflammatory properties. The quoted $p$ values for levels of statistical significance were two-sided. The attributable fraction, namely the percentage of all cases of ulcerative colitis attributed to the higher three intakes of dietary linoleic acid, was calculated using the formula, attributable fraction $=(O R$ $-1 / \mathrm{OR})^{*} \%$ cases in that quartile. The data were analysed by $\mathrm{A}$ Hart and R Luben using the STATA statistical package. A full cohort analysis was not used in this study as for some countries there were no regional or national databases of patients with inflammatory bowel disease; hence, here, case ascertainment may be incomplete. The hypothesis regarding linoleic acid, was an a priori one, developed approximately 2 years before the data on linoleic acid were analysed.

\section{RESULTS}

A total of 126 incident cases of ulcerative colitis were identified of which $47 \%$ were women. The age at diagnosis was known for 111 (88\%) cases with the median age being 60.0 years (range, $39.6-80.8$ years) with a median time between recruitment and

Table 1 Participating centres and characteristics of the cohorts

\begin{tabular}{|c|c|c|c|}
\hline Centre and country & Size of cohort & Nature of cohort & $\begin{array}{l}\text { No of participants } \\
\text { identified with incident } \\
\text { ulcerative colitis }\end{array}$ \\
\hline Norfolk, UK & 25639 & $\begin{array}{l}\text { Population-based cohort of men and women aged } \\
45-74 \text { years. Recruited between } 1993 \text { and 1997. Cases } \\
\text { identified from follow-up questionnaires, in-patient } \\
\text { admission data and histopathology records }\end{array}$ & 25 \\
\hline Heidelberg, Germany & 25540 & $\begin{array}{l}\text { Population-based cohort of men aged } 45-65 \text { years and } \\
\text { women aged } 35-65 \text { years. Recruited between } 1994 \text { and } \\
\text { 1998. Cases identified from follow-up questionnaires }\end{array}$ & 4 \\
\hline Potsdam, Germany & 27548 & $\begin{array}{l}\text { Population-based cohort, men and women, aged } \\
35-64 \text { years. Recruited between } 1994 \text { and } 1998 \text {. Cases } \\
\text { identified from follow-up questionnaires }\end{array}$ & 13 \\
\hline Florence, Italy & 13583 & $\begin{array}{l}\text { Population-based cohort, men and women aged } 34-64 \\
\text { years. Recruitment between } 1993 \text { and } 1998 \text {. Cases } \\
\text { identified from regional databases of inflammatory bowel } \\
\text { disease }\end{array}$ & 8 \\
\hline Umeå, Sweden & 25732 & $\begin{array}{l}\text { Population-based cohort, men and women aged } \\
30-60 \text { years. Recruited between } 1992 \text { and } 1996 \text {. Cases } \\
\text { identified from regional databases of inflammatory bowel } \\
\text { disease }\end{array}$ & 15 \\
\hline Malmö, Sweden & 28098 & $\begin{array}{l}\text { Population based cohort, men and women aged } 45-69 \\
\text { years. Recruited between years } 1991-1996 \text {. Cases } \\
\text { identified from regional databases of inflammatory bowel } \\
\text { disease }\end{array}$ & 21 \\
\hline $\begin{array}{l}\text { Aarhus and } \\
\text { Copenhagen, Denmark }\end{array}$ & 57053 & $\begin{array}{l}\text { Population-based cohort of men and women aged } \\
50-64 \text { years. Recruited between } 1993 \text { and } 1997 \text {. Cases } \\
\text { identified from national databases of inflammatory bowel } \\
\text { disease }\end{array}$ & 40 \\
\hline Total & 203193 & & 126 \\
\hline
\end{tabular}


diagnosis of 4.0 years (range, 1.7-11.3 years). For the cases, 79\% had the extent of their disease assessed by colonoscopy or barium enema, with $68 \%$ of these having left-sided disease up to the splenic flexure. The cases were age- and gender-matched with 504 controls from the centre in which the case was diagnosed. The completeness of the dietary data for the individual fatty acids was 100\% for controls and $99 \%$ for cases. In the cohort, no patients were vegetarians and only three $(0.6 \%)$ controls were. In this cohort, cigarette smoking at recruitment did not affect the risk of incident ulcerative colitis $(\mathrm{OR}=1.38,95 \% \mathrm{CI}=0.90$ to $2.12, \mathrm{p}=0.14)$. All seven centres supplied data on the dietary intake of linoleic, $\alpha$-linolenic and oleic acids. Information for eicosapentaenoic and docosahexaenoic acid was available for six centres, as the food frequency questionnaires have not been computed for these in one centre (Italy).

The highest quartile of intake of linoleic acid was associated with an increased risk of ulcerative colitis $(\mathrm{OR}=2.49,95 \%$ $\mathrm{CI}=1.23$ to $5.07, \mathrm{p}=0.01$ ) when adjusted for age at recruitment, gender, centre, energy intake and cigarette smoking (table 2). Similarly, there was a statistically significant trend across quartiles of linoleic acid intake $(\mathrm{OR}=1.32$ per quartile increase, $95 \% \mathrm{CI}=1.04$ to $1.66, \mathrm{p}=0.02)$. Furthermore, the effect of linoleic acid intake adjusted for aspirin use, for which data were available for $78.1 \%$ of subjects, still maintained the statistically significant effect. Similarly, adjusting for educational level did not affect the results for linoleic acid. The highest quartile of linoleic acid increased the risk of ulcerative colitis in both genders, which was statistically significant in women ( $\mathrm{OR}=3.47,95 \% \mathrm{CI}=1.13$ to $10.62, \mathrm{p}=0.03)$, but not men $(\mathrm{OR}=2.05,95 \% \mathrm{CI}=0.80$ to $5.26, \mathrm{p}=0.13)$. The results were similar if only cases were analysed, for whom details of the confirmatory investigations were supplied ( $87 \%$ of cases), (OR trend $=1.41,95 \% \mathrm{CI}=1.11$ to $1.80, \mathrm{p}=0.005)$. The attributable fraction for the highest three quartiles of intake of dietary linoleic acid was $30 \%$; ie, nearly a third of cases of ulcerative colitis could be attributed to the intake of that in the highest three quartiles. The effect of linoleic acid intake, adjusted for other fatty acids, for which complete data was available from six of the seven centres, showed a similar effect size to the previous analyses, although it was of borderline statistical significance (trend $\mathrm{OR}=1.28,95 \% \mathrm{CI}=0.97$ to $1.68, \mathrm{p}=0.08$ ).

The only other fatty acid for which an association was found with the development of ulcerative colitis was a negative one with an increasing dietary intake of the n-3 polyunsaturated fatty acid, docosahexaenoic acid, when adjusted for the other nutrients. For the highest quartile, the odds ratio was 0.23 (95\% $\mathrm{CI}=0.06$ to 0.97$)$ with a significant negative trend across quartiles (OR for trend $=0.59,95 \% \mathrm{CI}=0.37$ to $0.94, \mathrm{p}=0.03$ ).

\section{DISCUSSION}

The main finding of this study was more than a doubling of the risk of ulcerative colitis with the highest intake of the dietary $n-6$ PUFA, linoleic acid. If the association is causative then $30 \%$ of all cases could be attributed to such higher intakes. This finding is supported by plausible biological mechanisms. n-6 PUFAs are present in colonocyte membranes as arachidonic acid ( $n-6$ derived) which can be released from cell membranes and metabolised to prostaglandins, leukotrienes and thromboxanes. These have immuno-stimulatory effects ${ }^{14-16}$ and may predispose to the development of ulcerative colitis. The metabolites of arachidonic acid, including prostaglandin $\mathrm{E}_{2}$, leukotriene $\mathrm{B}_{4}$ and thromboxane $\mathrm{A}_{2}$, have pro-inflammatory effects including aggregation, chemotaxis and release of lysosomal enzymes from neutrophils. ${ }^{14-16}$
Thromboxanes have many actions including activation of neutrophils, ${ }^{17}$ production of leukotriene $\mathrm{B}_{4}{ }^{18}$ and modulation of T cell function. ${ }^{19}$ A high dietary intake of $n-6$ PUFAs and their incorporation into colonic cell membranes would lead to a source of pro-inflammatory molecules which could pre-dispose to ulcerative colitis. The essential n-6 PUFA fatty acid, linoleic acid, is present in red meat (particularly beef and pork), cooking oils (especially corn and sunflower oils) and polyunsaturated margarines. Foods containing lower amounts of linoleic acid are fish, white meats, olive oil, butter and non-polyunsaturated margarines. Linoleic acid undergoes carbon chain elongation and desaturation to form arachidonic acid which is incorporated into cell membranes. Increased levels of arachidonic acid and its metabolites, prostaglandin $\mathrm{E}_{2}$, leukotriene $\mathrm{B}_{4}$ and thromboxane $\mathrm{B}_{2}$, are found in the colonic mucosa of patients with ulcerative colitis. $^{24520}$ The degree of inflammatory cell infiltrate also correlates with the mucosal arachidonic acid concentration $(r=0.89, p<0.05)$ in humans. ${ }^{2}$ Finally, the first-line drugs used to treat ulcerative colitis, namely 5 -aminosalicyclic compounds, suppress prostaglandin $\mathrm{E}_{2}$ levels in a dose-dependent manner. ${ }^{4}$ The positive association with linoleic acid was unlikely to be a confounding effect of the other PUFAs, as adjustment for these, did not alter the magnitude of the odds ratio. However, the $p$ value changed from a significant $(p=0.02)$ to a borderline significant effect $(p=0.08)$ which may be due to a reduced sample size for this particular analysis, as data on certain n-3 PUFAs were not available from one centre. Furthermore, the intake of linoleic acid is likely to be lower in this southern European centre than northern regions, making any true differences more difficult to detect.

This investigation also found a statistically significant negative association with an increasing dietary intake of docosahexaenoic acid, when adjusted for the other fatty acids which can affect its metabolism. The highest dietary intake of this n-3 PUFA was associated with a $77 \%(95 \% \mathrm{CI}=3 \%$ to $94 \%)$ reduction in the chances of developing ulcerative colitis. There are several plausible biological mechanisms to support how, through its anti-inflammatory properties, docosahexaenoic may prevent colonic inflammation. These include promoting the release of phospholipases $\mathrm{D}$ from membranes, which leads to the activation of its anti-proliferative effects in lymphoid cells. ${ }^{21}$ The nutrients also inhibit protein kinase $C,{ }^{22}{ }^{23}$ which decreases the levels of secondary messengers involved in inflammation. Finally, they may have a direct action on gene expression for inflammatory mediators, as fish oil has such effects on several tissues, including spleen lymphocytes. ${ }^{24}$ The independent effects of linoleic and docosahexaenoic acid make confounding by social class less likely, as both may be associated with higher social classes.

The effect of total dietary polyunsaturated fatty acids on the risk of developing incident ulcerative colitis has been investigated in only a few epidemiological studies. ${ }^{6-8} 25$ In the only prospective investigation, again in this EPIC cohort, a marginally significant positive association was reported with an increasing percentage intake of energy from the total of all polyunsaturated fatty acids (trend across quartiles $\mathrm{OR}=1.19$ (95\% CI $=0.99$ to 1.43 ) $p=0.067){ }^{7}$ Three case-control studies from Israel, Holland and Japan have investigated total PUFAs, ${ }^{4}{ }^{25}$ although by the nature of this study design, recall bias may exist. The investigation from Israel of 54 patients with ulcerative colitis, most of whom were diagnosed 3-4 months before interview, reported that a high intake of total polyunsaturated fatty acids increased the risk of ulcerative colitis by 6.54 times $(95 \% \mathrm{CI}=1.45$ to 29.68$) .{ }^{8}$ Similar results were 
Table 2 Odds ratios for developing ulcerative colitis by quartile of intake of specific polyunsaturated fatty acids (PUFAs)

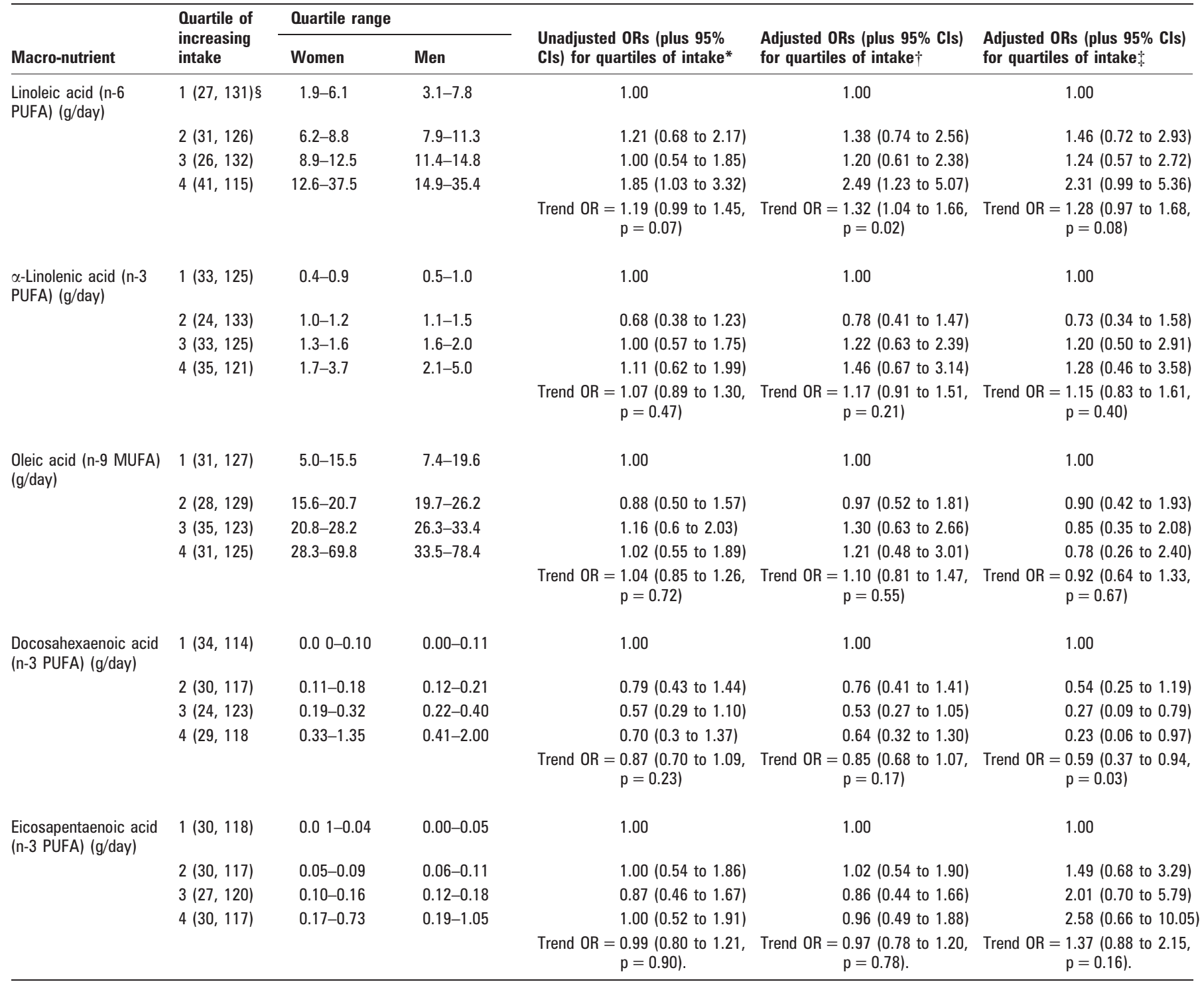

*0dds ratios (ORs) adjusted for age at recruitment into the European Prospective Investigation into Cancer and Nutrition (EPIC), gender and centre.

†Odds ratios adjusted for age at recruitment into EPIC, gender, centre, cigarette smoking and total energy intake.

\$Odds ratios adjusted for age at recruitment into EPIC, gender, centre, cigarette smoking, total energy intake and the other listed fatty acids.

$\S$ Results in parentheses are number of cases and number of controls.

$\mathrm{Cl}$, confidence interval; MUFA, mono-unsaturated fatty acid.

reported in a case-control study of 43 patients from Holland, all diagnosed within 5 months of recruitment, which detected an odds ratio of 5.1 (95\% CI 1.0 to 26.7) for the highest versus lowest tertile of intake. ${ }^{6}$ The Dutch study was the only one to specifically assess linoleic acid intake and reported that the two higher tertiles doubled the risk of disease, although these did not reach statistical significance. In both these investigations, the recall of pre-symptomatic diet was likely to be accurate, as the time interval between diagnosis and recruitment was short. The Japanese case-control study found no statistically significant differences for total PUFAs and n-6 PUFAs, although it is possible that the dietary information was less accurate as patients had to remember their pre-symptomatic diet up to 3 years previously. ${ }^{25}$ There is also support for the fatty acid hypothesis from clinical studies in patients with established ulcerative colitis. The arachidonic acid composition of phospholipids in the colonic mucosa was found to be higher in patients than in healthy controls $(12.5 \mathrm{~mol} \%$ vs $6.8 \mathrm{~mol} \%$, $\mathrm{p}<0.001) .^{2}$ Furthermore, the amount of inflammatory exudate correlated with the arachidonic acid composition of phospholipids in the colonic mucosa $(r=0.89, p<0.005)$. Other clinical studies have reported significantly higher concentrations of arachidonic and linoleic acid in the plasma ${ }^{26}$ and neutrophil phospholipids ${ }^{27}$ in patients with ulcerative colitis than controls. Data collected from this EPIC cohort have previously been assessed for the effect of other general nutrients and certain vitamins and minerals on the risk of developing ulcerative colitis and no associations were reported. ${ }^{7}$

The principal advantage of this prospective cohort investigation over previous case-control studies was the minimisation of recall biases, as the dietary information was recorded prior to the diagnosis of the disease. Also, this design reduced the selection biases inherent in retrospective case-control studies, as cases and controls were prospectively ascertained from the same, and a large, baseline population. Random measurement error in the food frequency questionnaire will result in an under- 
estimation of the odds ratios for dietary constituents rather than produce spurious positive associations. A more accurate method of assessing diet is weighed records of food intake, but these are impractical to use in large-scale epidemiological studies. A validation study, conducted in EPIC-Norfolk, UK, comparing dietary intakes between 16-day weighed records and food frequency questionnaires reported correlation coefficients between 0.39 and 0.90 for dietary variables. ${ }^{28}$ Of particular relevance to the findings of this study was a Spearman correlation coefficient of 0.64 for fat intake. A limitation of our methodology was that we only had smoking data at recruitment and not during subsequent follow-up. Ulcerative colitis may be more common in those who cease smoking. The generalisability of any cohort study, namely its external validity, needs to be considered. The population in this investigation was predominantly middle-aged to elderly, whereas ulcerative colitis is traditionally regarded as a disease presenting in earlier life. However, a large incidence study of patients with ulcerative colitis, treated in centres across Europe, showed that the age at diagnosis was similar across all ages in men, but in women the incidence declined with increasing age. ${ }^{29}$ Therefore, our study had an under-representation of younger women with ulcerative colitis. The disease distribution was similar in this cohort compared with the European incidence study (32\% vs $21 \%$ for disease proximal to the splenic flexure). ${ }^{30}$ Finally, data from a UK population showed the dietary intake of $\mathrm{n}-6$ PUFAs is similar across adult age ranges. ${ }^{31}$ Although we did not detect a negative association with cigarette smoking at recruitment, this may be because healthier volunteers are more likely to participate in a cohort study who are less likely to smoke.

The findings from aetiological studies may help identify potential new treatments for patients with inflammatory bowel disease. There are no dietary modifications of benefit in patients with ulcerative colitis, although, based on this study's findings, a diet low in linoleic acid may merit investigation. In patients with Crohn's disease, enteral diets can induce remission and, in these, the fat content may be important. In one trial, 62 patients with active Crohn's disease were randomised to receive either a diet high in oleate and low in linoleate, a diet high in linoleate and low in oleate, or oral prednisolone. ${ }^{32}$ At 4 weeks, the diet high in linoleate resulted in a higher remission rate than that with the lower content $(63 \%$ vs $27 \%, p=0.008)$. These findings are surprising as it was expected that linoleate, through its conversion to pro-inflammatory eicosonoids, would stimulate inflammation. The reasons for this finding are unknown, although possibilities are the relative proportions of other fatty acids and the quantitative amounts. Clinical studies which have compared the percentage of linoleic in plasma between patients with Crohn's disease and controls have either reported no difference $e^{2633} 34$ or lower amounts. ${ }^{35}$

In summary, this prospective cohort study found that a high dietary intake of the n-6 PUFA linoleic acid more than doubled the risk of developing incident ulcerative colitis. Measurement error in the food frequency questionnaire means the size of this reported effect is probably an under-estimate of the true effect. The positive association may reflect a causal association because of both a plausible biological mechanism and supportive evidence from other epidemiological studies. The association needs to be further investigated in other aetiological work in different populations to assess consistency. If the positive association is causal, then there is substantial potential for reducing the incidence of ulcerative colitis through dietary modification.
Acknowledgements: The authors greatly acknowledge the residents in each centre who kindly participated in the study.

Funding: This analysis was funded by The Sir Halley Stewart Trust, The National Association for Colitis and Crohn's Disease and The NHS Executive Eastern Region. EPIC-Norfolk is supported by Cancer Research UK and The Medical Research Council, UK. EPIC-Malmö is supported by The Swedish Cancer Society, The Swedish Research Council and The Region of Skane. EPIC-Denmark is supported by The Danish Cancer Society. EPIC-Heidelberg is supported by "Stiftung Landesbank Baden-Württemberg", the European Union and Deutsche Krebshilfe. EPIC-Potsdam is supported by the Federal Ministry of Research and Technology, the European Union and Deutsche Krebshilfe. EPIC-Florence is supported by the Associazione Italiana per la Ricerca contro il Cancro (AIRC-Milan) and Regione Toscana.

\section{Competing interests: None.}

Ethics approval: The names and locations of the ethics committees that approved the collection of data were: Norwich District Ethics Committee, UK; Ethical Committee of the Medical Faculty at the University of Heidelberg, Germany; Regionala etikprövningsnämnden i Umeå, Sweden; De Videnskabsetiske Komitéer for Region Hovedstaden, Regionsgården, Denmark; Comitato Etico Locale dell 'Azienda Sanitaria di Firenze, Italy; Ethics Committee at The Medical Association of the State of Brandenburg in Cottbus, Germany; and Lund University Ethics Committee, Sweden.

\section{List of collaborators and authors}

Denmark

A Tjonneland (Head of Department) and A Olsen (Post-doctoral Researcher), Institute of Cancer Epidemiology, Danish Cancer Society,

Copenhagen, Denmark

K Overvad (Professor of Clinical Epidemiology),

Department of Clinical Epidemiology, Aalborg Hospital,

Aarhus University Hospital, Aalborg, Denmark

Germany

Potsdam

MM Bergmann (Scientist) and H Boeing (Head of the Department of Epidemiology), Department of Epidemiology, German Institute of Human Nutrition,

Potsdam-Rehbrücke, Nuthetal, Potsdam, Germany

Heidelberg

G Nagel (Senior Researcher),

Division of Clinical Epidemiology,

DKFZ-Heidelberg, Heidelberg, Germany:

and Institute of Epidemiology, Ulm University,

Ulm, Germany

$\mathrm{J}$ Linseisen (Principal Investigator EPIC-Heidelberg),

Division of Clinical Epidemiology, Division of Clinical Epidemiology,

DKFZ-Heidelberg, Heidelberg, Germany

Sweden

Umeå

G Hallmans (Professor of Nutritional Research) and $\AA$ Danielsson (Professor of

Gastroenterology),

Department of Public Health \& Clinical Medicine

Umeå University, Umeå, Sweden

R Palmqvist (Senior Lecturer),

Department of Medical Biosciences, Pathology,

Umeå University, Umeå, Sweden

H Sjodin (Statistician Medical Biobank),

Umeå University Hospital, Umeå, Sweden

G Hägglund (Research Assistant),

Department of Nutritional Research,

Umeå University, Umeå, Sweden

Malmö

G Berglund (Professor of Medicine), S Lindgren (Professor of Gastroenterology) and 0 Grip (Consultant Gastroenterologist),

Department of Clinical Sciences, University Hospital MAS,

Malmö, Sweden

Italy

D Palli (Head of Department) and G Masala (Research Scientist),

Molecular and Nutritional Epidemiology Unit,

Cancer Research and Prevention Centre - ISPO,

Florence, Italy

United Kingdom 
NE Day (Emeritus Professor of Epidemiology), R Luben (Head of Bioinformatics), and A Welch (Former Head Research Nutritionist),

Strangeways Research Laboratory, Cambridge, UK

K-T Khaw (Professor of Clinical Gerontology),

Clinical Gerontology Unit, Addenbrooke's Hospital,

Cambridge, UK

S Bingham (Group Leader, Diet and Cancer),

MRC Dunn Human Nutrition Unit,

Cambridge, UK

E Riboli (Head, Division of Epidemiology, Public Health and Primary Care),

Faculty of Medicine, Imperial College London,

London, UK

H Kennedy (Consultant Gastroenterologist),

Norfolk \& Norwich University Hospital NHS Trust,

Norwich, UK

A Hart (Senior Lecturer in Gastroenterology),

University of East Anglia,

Norwich, UK

Provenance and peer review: Not commissioned; externally peer reviewed.

\section{REFERENCES}

1. Eaden JA, Abrams KR, Mayberry JF. The risk of colorectal cancer in ulcerative colitis: a meta-analysis. Gut 2001;48:526-35.

2. Nishida T, Miwa H, Shigematsu A, et al. Increased arachidonic acid composition of phospholipids in colonic mucosa from patients with active ulcerative colitis. Gut 1987;28:1002-7.

3. Rampton D, Collins C. Review article: thromboxanes in inflammatory bowel disease - pathogenic and therapeutic implications. Aliment Pharmacol Ther 1993:7:357-67.

4. Sharon $\mathbf{P}$, Ligumsky $M$, Rachilewitz $\mathrm{D}$, et al. Role of prostaglandins in ulcerative colitis. Enhanced production during active disease and inhibition by sulfasalazine. Gastroenterology 1978;75:638-40.

5. Sharon P, Stenson W. Enhanced synthesis of leukotriene B4 by colonic mucosa in inflammatory bowel disease. Gastroenterology 1984;86:453-60.

6. Geerling BJ, Dagnelie PC, Badart-Smook A, et al. Diet as a risk factor for the development of ulcerative colitis. Am J Gastroenterol 2000;95:1008-13.

7. Hart A, Luben R, Olsen A, et al. Diet in the aetiology of ulcerative colitis - A European prospective cohort study. Digestion 2008;77:57-64.

8. Reif S, Klein I, Lubin F, et al. Pre-illness dietary factors in inflammatory bowel disease. Gut 1997:40:754-60.

9. Riboli E. Nutrition and cancer: background and rationale of the European Prospective Investigation into Cancer and Nutrition. Ann Oncol 1992;3:783-91.

10. Kaaks R, Slimani N, Riboli E. Pilot phase studies on the accuracy of dietary intake measurements in the EPIC project: overall evaluation of results. European Prospective Investigation into Cancer and Nutrition. Int J Epidemiol 1997;26:S26-36.

11. Margetts BM, Pietinen P. European Prospective Investigation into Cancer and Nutrition: Validity studies on dietary assessment methods. Int J Epidemiol 1997;26:S1-5.

12. Calkins BM. A meta-analysis of the role of smoking in inflammatory bowel disease. Dig Dis Sci 1989;34:1841-54.

13. Logan R, Kay CR. Oral contraception, smoking and inflammatory bowel disease findings in the Royal College of General Practitioners Oral Contraception Study. Int J Epidemiol 1989;18:105-7.

14. Goldman DW, Pickett WC, Goetzl EJ. Human neutrophil chemotactic and degranulating activities of leukotriene $\mathrm{B}_{5}\left(\mathrm{LTB}_{5}\right)$ derived from eicosapentaenoic acid. Biochem Biophys Res Comm 1983;117:282-88.

15. Heller A, Koch T, Schmeck J, et al. Lipid mediators in inflammatory disorders. Drugs 1998:55:487-96.
16. Lee TH, Mencia-Huerta JM, Shih C, et al. Characterization and biological properties of 5,12-dihydroxy derivatives of eicosapentaenoic acid, including leukotriene B5 and the double lipoxygenase product. J Biol Chem 1984;259:2383-9.

17. Kitchen $\mathbf{E}$, Boot JR, Dawson W. Chemotactic activity of thromboxane B2, prostaglandins and their metabolites for polymorponuclear leucocytes. Prostaglandins 1978;16:239-44.

18. Goldman G, Welbourn R, Valeri C, et al. Thromboxane A2 induces leukotriene B4 synthesis that in turn mediates neutrophil diapedesis via CD18 activation. MicrovasC Res 1991:41:367-75.

19. Kelly JP, Johnson MC, Parker CW. Effect of inhibitors of arachidonic acid metabolism on mitogenesis in human lymphocytes: possible role of thromboxanes and products of the lipoxygenase pathway. J Immunol 1979;122:1563-71

20. Boughton-Smith N, Hawkey C, Whittle B. Biosynthesis of lipoxygenase and cyclooxygenase products from $\left[{ }^{14} \mathrm{C}\right]$-arachidonic acid by human colonic mucosa. Gut 1983:24:1176-82.

21. Diaz 0, Berquand $A$, Dubois $M$, et al. The mechanism of docosahexaenoic acidinduced phospholipase $D$ activation in human lymphocytes involves exclusion of the enzyme from lipid rafts. J Biol Chem 2002;277:368-78.

22. May C, Southworth A, Calder PC. Inhibition of lymphocyte protein kinase $\mathrm{C}$ by unsaturated fatty acids. Biochem Biophys Res Commun 1993;195:823-8.

23. Speizer L, Watson M, Brunton L. Differential effects of omega-3 fish oils on protein kinase activities in vitro. Am J Physiol 1991;261:E109-14.

24. Robinson D, Urakaze M, Huang R, et al. Dietary marine lipids suppress continuous expression of interleukin-1 beta gene transcription. Lipids 1996;31:S23-S31.

25. Sakamoto N, Kono S, Wakai K, et al. Dietary risk factors for inflammatory bowel disease: a multi-centre case-control study in Japan. Inflammatory bowel diseases 2004:11:154-63.

26. Esteve-Comas M, Nunez M, Fernandez-Banares F, et al. Abnormal plasma polyunsaturated fatty acid pattern in non-active inflammatory bowel disease. Gut 1993;34:1370-3.

27. Kawakami Y, Okada H, Murakami Y, et al. Dietary intake, neutrophil fatty acid profile, serum antioxidant vitamins and oxygen radical absorbance capacity in patients with ulcerative colitis. J Nutr Sci Vitaminol 2007;53:153-9.

28. Bingham SA, Gill C, Welch A, et al. Validation of dietary assessment methods in the UK arm of EPIC using weighed records and 24 hour urinary nitrogen and potassium and serum vitamin C and carotenoids as biomarkers. Int J Epidemiol 1997;26(Suppl 1):S137-51.

29. Shivananda S, Lennard-Jones JE, Logan R, et al. Incidence of inflammatory bowel disease across Europe: is there a difference between north and south? Results of the European collaborative study on inflammatory bowel disease (EC-IBD). Gut 1996;39:690-7.

30. Lennard-Jones JE, Shivananda S. Clinical uniformity of inflammatory bowel disease at presentation and during the first year of disease in the north and south of Europe. EC-IBD Study group. Eur J Gastroenterol Hepatol 1997;9:353-9.

31. Henderson L, Gregory J, Irving K, et al. The National Diet and Nutrition Survey: adults aged 19-64 years. Office for National Statistics, Food Standards Agency 2003:2:67-76.

32. Gassull M, Fernandez-Banares F, Cabre E, et al. Fat composition may be a clue to explain the primary therapeutic effect of enteral nutrition in Crohn's disease: results of a double blind randomised multicentre European trial. Gut 2002; 51:164-8

33. Esteve-Comas $\mathbf{M}$, Ramirez M, Fernandez-Banares F, et al. Plasma polyunsaturated fatty acid pattern in active inflammatory bowel disease. Gut 1992;33:1365-9.

34. Geerling BJ, Houwelingen MD, Badart-Smook A, et al. Fat intake and fatty acid profile in plasma phospholipids and adipose tissue in patients with Crohn's disease, compared with controls. Am J Gastroenterol 1999;94:410-7.

35. Siguel $\mathbf{E}$, Lerman R. Prevalence of essential fatty acid deficiency in patients with chronic gastrointestinal disorders. Metabolism 1996:45:12-23. 